CERN-PPE/93-65

15 April 1993

\title{
Neutrino Electron Scattering
}

\author{
Gaby Rädel ${ }^{\dagger}$ and Rolf Beyer ${ }^{\S}$ \\ $\dagger$ DESY, Hamburg, Germany \\ $\S$ CERN, Geneva, Switzerland.
}

(to appear in Mod. Phys. Lett. A)

\begin{abstract}
This article reviews experimental results obtained from studies of neutrino electron scattering and shows in particular the important input from these experiments to the improved knowledge of weak neutral currents and the confirmation of the Standard Model at tree level. Special emphasis is put on recent high precision $\nu$ e-experiments, whose results on electroweak parameters allow, in combination with precise results obtained at higher $Q^{2}$, a test of the Standard Model at the level of higher order corrections.
\end{abstract}




\title{
Neutrino Electron Scattering
}

\author{
Gaby RÄDeL and Rolf Beyer
}

\section{Introduction}

The first observation of a handful of $\bar{\nu}_{\mu} e$-scattering events by the Gargamelle bubble chamber [1] in 1973 was a turning point in elementary particle physics. The existence of weak neutral currents was demonstrated. A phenomenon which could not be explained by the long-standing Fermi-type theory of weak interactions but was predicted by a combined electroweak theory, formulated by Glashow, Weinberg, and Salam [2]: the so-called 'Standard Model' of electroweak interactions. Since then several experiments with the aim of studying the elastic scattering of neutrinos on electrons have been performed, all of which added further support to the Standard Model.

The first generation of $\nu$-experiments, performed in the 1970's, were experiments dedicated to the confirmation of the Standard Model at tree level. Integral cross sections and first values for the electroweak mixing angle, $\sin ^{2} \theta_{W}$, were obtained. These early experiments were either performed at accelerators in $\nu_{\mu}$ or $\bar{\nu}_{\mu}$ beams or at nuclear reactors, where $\bar{\nu}_{e} e$ scattering was observed for the first time. However, all these experiments suffered from rather poor statistics, due to the very low cross section of $\nu$ e scattering. In 1980 the number of observed events by all experiments was in the order of 100 .

The situation changed in the 1980's with the second generation of experiments. They were counter experiments with large target mass dedicated neutrino detectors utilizing high intensity neutrino beams. The statistics collected were an order of magnitude higher than for the previous generation, thus making it possible to measure kinematical distributions and differential cross sections. With the last experiment in this series (CHARM II [3]) electroweak parameters were measured to such an accuracy that testing the Standard Model at higher orders became possible. In addition limits on quantities beyond the Standard Model could be derived. At last neutrino electron scattering nowadays is used even as an experimental tool to detect solar neutrinos [4], thus testing the Standard Solar Model.

From a theoretical point of view the process of $\nu$ e-scattering is comparatively simple. In the first, theoretical, section of this article we define the relevant quantities to describe $\nu e$-scattering in the theoretical frame, of the Standard Model at tree level. Going to higher orders we make some remarks on radiative corrections as well as physics beyond the Standard Model.

On the other hand, experimentally $\nu e$-scattering is a very difficult task due the smallness of its cross section $\left(\sim 10^{-42} \mathrm{~cm}^{-2}\right)$ and to the presence of abundant competing processes which cannot be totally eliminated on an event by event basis. Therefore sophisticated experimental methods have been invented, whose realization we describe in the more detailed second section. For each method a selected experiment is described in detail and its results are presented.

Finally we combine the results of all $\nu e$-experiments and compare them to the results of other measurements and to the predictions of the Standard Model.

\section{Some Theoretical Remarks}

Neutrino-electron scattering is a purely leptonic process where a neutrino scatters off an electron by the exchange of a virtual vector boson (fig. 1). The $Q^{2}$ range covered at present by neutrino electron scattering experiments reaches from $10^{-6}$ at nuclear reactors to $10^{-2}$ at accelerators but is always small compared to the mass of the $Z^{0}$. 


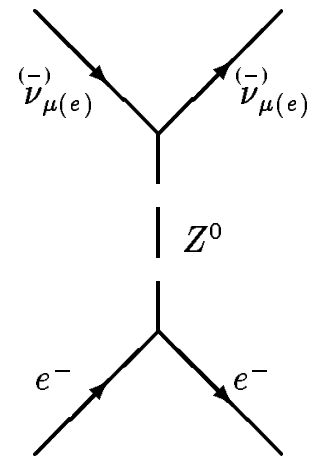

(a)

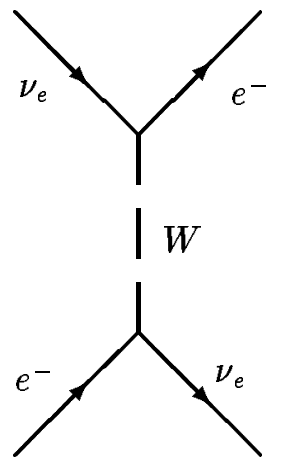

(b)

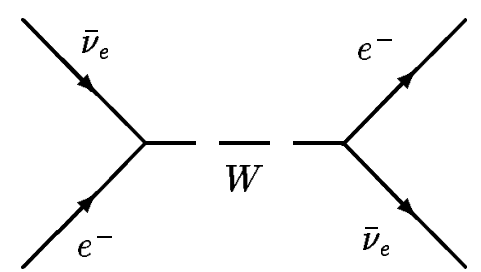

(c)

Figure 1: Feynman diagrams for the processes of neutral current (NC) ve-scattering (a), and charged current $(C C) \nu_{e} e$-scattering via the exchange of a $W$-Boson $(b, c)$.

\section{Kinematics}

The kinematics of elastic neutrino electron scattering is fully described by a single variable; for instance by $\theta_{e}$, the angle of the outgoing electron with respect to the neutrino beam. Let $E_{\nu}$ and $E_{e}$ be the energies of the incoming neutrino and outgoing electron respectively, $m_{e}$ the electron mass, and $y=E_{e} / E_{\nu}$ be the fractional energy loss of the neutrino in the laboratory system. With the assumption of $m_{e} \ll E_{e}$ and the small angle approximation for $\cos \theta_{e}$ one finds

$$
E_{e} \theta_{e}^{2}=2 m_{e}(1-y)
$$

and, as $0 \leq y \leq 1$, the experimentally important constraint: $E_{e} \theta_{e}^{2}<2 m_{e}$.

This means the outgoing electron is scattered in extremely forward direction, which is used experimentally to subtract, on a statistical basis, background events, which have a much broader distribution in $E_{e} \theta_{e}^{2}$. On the other hand this signature makes it impossible to measure directly the $y$-distribution of $\nu e$-reactions, as reachable resolutions are just in the same order of magnitude as the kinematical bound.

\section{Cross sections}

The model independent effective neutral current interaction Lagrangian can be written as [5]:

$$
\mathcal{L}_{\text {eff }}^{N C}=2 \sqrt{2} G_{F}\left(\bar{\nu}_{L} \gamma^{\alpha} \nu_{L}\right)\left(g_{L} \bar{e}_{L} \gamma_{\alpha} e_{L}+g_{R} \bar{e}_{R} \gamma_{\alpha} e_{R}\right)+\text { h.c. }
$$

In the limit of small $Q^{2}$, propagator effects can be neglected. $L, R$ denote the chirality of the fermion. The same can be expressed in terms of the axialvector and vector couplings of the neutral vector boson to the electron. The relation between the chiral couplings and $g_{V}^{e}$ and $g_{A}^{e}$ is given by

$$
2 g_{L}=g_{V}+g_{A} \quad \text { and } \quad 2 g_{R}=g_{V}-g_{A}
$$

From the effective Lagrangian the differential cross section - often called $y$-distribution - is readily calculable:

$$
\frac{\mathrm{d} \sigma^{\nu_{\mu}, \bar{\nu}_{\mu}}}{\mathrm{d} y}=\frac{2 G_{F}^{2} m_{e}}{\pi} E_{\nu}\left[g_{L, R}^{2}+g_{R, L}^{2}(1-y)^{2}-g_{L} g_{R} \frac{m_{e} y}{E_{\nu}}\right] .
$$

The $(1-y)^{2}$-term originates from angular momentum conservation, and suppresses backward scattering $(y=1)$ of (anti)neutrinos off (left-)right-handed electrons. For high neutrino energies $\left(E_{\nu} \gg m_{e}\right)$ the term linear in $y$ is negligible. 

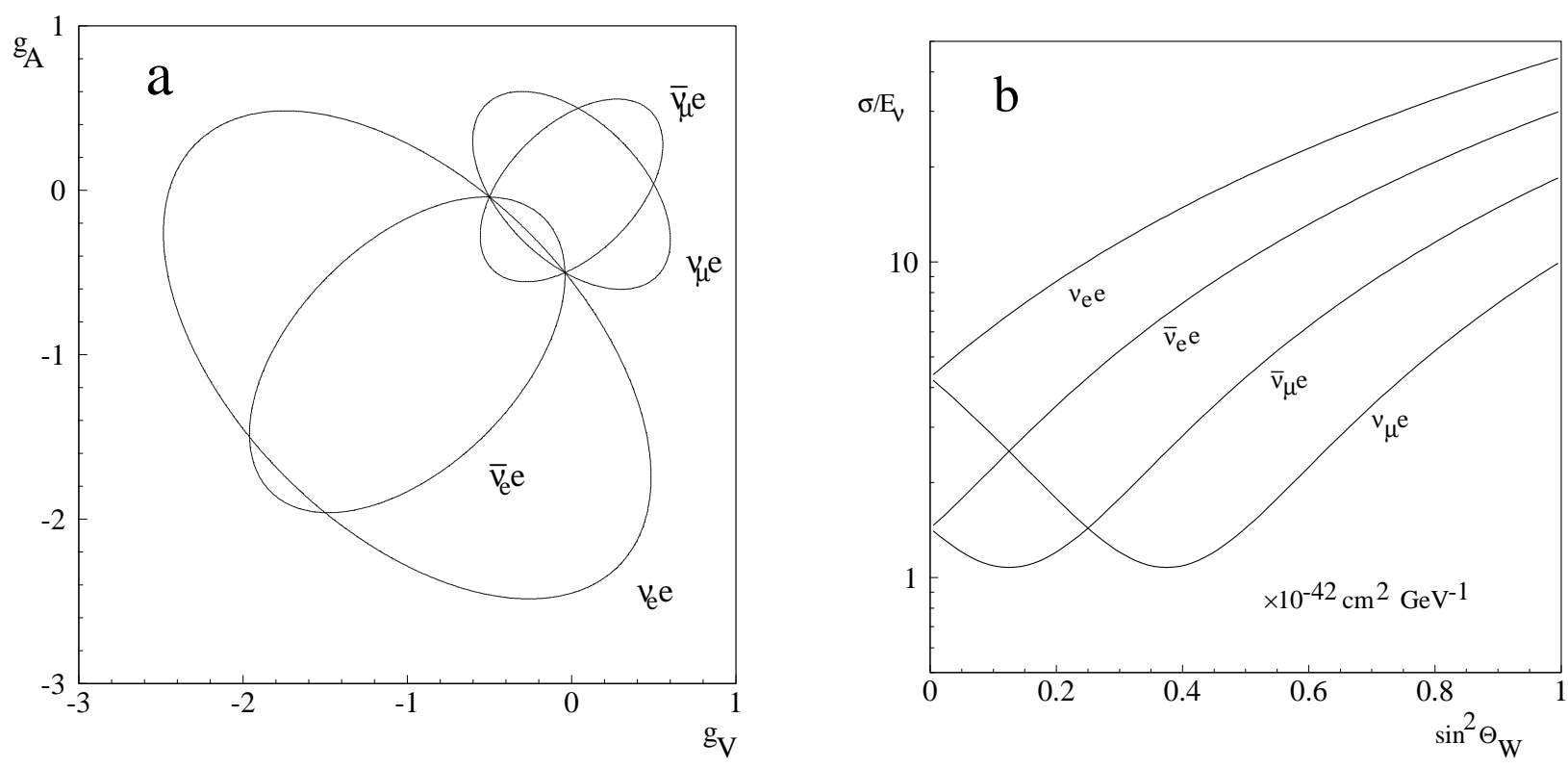

Figure 2: Dependence of neutrino-electron cross section on electroweak parameters. a): contours in the $g_{V}-g_{A}$ plane. The contours correspond to four ideal measurements with $\sin ^{2} \theta_{W}=0.23$. $b$ ): Cross section as a function of $\sin ^{2} \theta_{W}$. Note the logarithmic scale.

To obtain the cross section of $\nu_{e}$-scattering, where the charged current interaction contributes to the scattering amplitude, one has simply to substitute $g_{L}$ by $g_{L}+1$.

The Standard Model relates the coupling constants and the electroweak mixing angle $\sin ^{2} \theta_{W}$ and the relative coupling strength $\rho$ of the neutral current (NC) with respect to the charged current $(\mathrm{CC})$ :

$$
g_{R}=\rho \sin ^{2} \theta_{W} \quad \text { and } \quad g_{L}=\rho\left(\sin ^{2} \theta_{W}-1 / 2\right) .
$$

The resulting cross section dependence on $\sin ^{2} \theta_{W}$ is shown in fig. $2 \mathrm{~b}$. Obviously the neutral current coupling constants cannot be determined from a single measurement. Even a measurement of $\sigma\left(\nu_{\mu} e\right)$ and $\sigma\left(\bar{\nu}_{\mu} e\right)$ leaves a fourfold ambiguity for the coupling constants. A measurement of electron-neutrino electron scattering cross sections only partly resolves the problem. A twofold ambiguity remains. This problem can be solved using measurements fo the forward-backward asymmetry in $e^{+} e^{-}$-collisions. These experiments yield $g_{V}^{2} \ll g_{A}^{2}[6]$.

\section{Radiative corrections}

When considered in the context of the electroweak perturbation theory, the expression for the cross sections (4) is only true in first order of the coupling constant, i.e. in Born approximation (tree level). Experiments have, however, achieved an accuracy that makes it necessary to consider also contributions of higher order in the perturbation series (radiative corrections). Including corrections proportional to $G_{F} \alpha$ in the matrix elements, the differential cross section of neutrino-electron scattering (one-loop cross section) can be written as [7]:

$$
\frac{\mathrm{d} \sigma_{\nu e}}{\mathrm{~d} y}=\frac{\mathrm{d} \sigma_{\nu e}^{e w}}{\mathrm{~d} y}+\frac{\mathrm{d} \sigma_{\nu e}^{Q E D}}{\mathrm{~d} y} .
$$

The second term corresponds to pure QED corrections, while the first term is obtained from the tree-level result by replacing:

$$
G_{F} \rightarrow G_{F} \rho_{\nu e}\left(Q^{2}\right) \text { and } \sin ^{2} \theta_{W} \rightarrow \kappa^{\nu e}\left(Q^{2}\right) \sin ^{2} \theta_{W} .
$$


$\kappa^{\nu e}\left(Q^{2}\right)$ and $\rho_{\nu e}\left(Q^{2}\right)$ are correction functions which depend on the unknown parameters of the Standard Model, like the mass of the top quark $m_{t}$, and on the renormalization scheme applied. Neutrinoelectron scattering experiments extract results on $\sin ^{2} \theta_{W}$ from the tree-level formula, yielding so-called effective values $\left(\sin _{\nu e}^{2} \theta\right)$. To perform comparisons with results of other experiments one has to correct both results according to the same renormalization scheme. Therefore the choice of the renormalization scheme, and thus the definition of $\sin ^{2} \theta_{W}$ becomes important. One possibility is the $\overline{M S}$ scheme [8], where $\sin ^{2} \theta_{W}$ is defined as the ratio of the coupling constants $\sin ^{2} \bar{\theta}_{W}=e^{2}\left(Q^{2}\right) / g^{2}\left(Q^{2}\right)$.

A consequence of the QED term in (6) is that the $y$-distribution is changed according to the experimental method of its measurement. If the energy carried by external bremsstrahlung photons is not distinguished form the energy of the scattered electron, such as in the case of calorimetric detectors, the correction amounts to a few percent only [9]. In bubble chamber experiments however, where cuts are applied on additional vertex activity, QED effects might result in larger corrections. It is therefore essential that experimental results are corrected for all possible higher order electromagnetic effects.

\section{Beyond the Standard Model}

Inside the Standard Model a magnetic moment of the neutrino is forbidden. However, its possible existence has been discussed my many authors $[10,11]$. A $\nu e$-scattering process via a magnetic moment would change the helicity of the neutrino and contribute a non-coherent part to the cross section [10]:

$$
\frac{\mathrm{d} \sigma^{\mu}}{\mathrm{d} y}=\mu_{\nu}^{2} \mu_{B o h r}^{2} \frac{1-y}{y} .
$$

Limits on the magnetic moment can be derived by comparing cross section measurements of $\nu e-$ scattering to measurements were no neutrinos are involved. Due to the rise of (7) for low neutrino energies (the expression is not divergent due to a lower kinematic bound of the electron energy), the experimental limits are the better the lower the low-energy cut-off relative to the neutrino energy is. The integral cross section rises only logarithmically with the neutrino energy, so that possible magnetic moment effects are to be detected more easily at low energy neutrino sources.

A non-zero charge radius of the neutrino, on the other hand, does not change the spin of the neutrino and an additional term to the cross section would be added coherently. As a matter of fact, higher order vertex corrections, inside the Standard Model, do introduce a charge radius in the order of $\left\langle r^{2}\right\rangle \approx \mathcal{O}\left(10^{-34} \mathrm{~cm}^{2}\right)[12]$.

An anomoulus charge radius has the effect of modifying the vector part of the interaction and manifests itself in a change of the effective $\sin ^{2} \theta_{W}[13]$ :

$$
g_{V} \rightarrow-\frac{1}{2}+2\left(\sin ^{2} \theta_{W}+\delta\right) \quad \text { with } \quad \delta=\frac{\sqrt{2} \pi \alpha}{3 G_{F}}\left\langle r^{2}\right\rangle_{\text {anom }} .
$$

Limits for the charge radius can be obtained comparing $\sin ^{2} \theta_{W}$ obtained from neutrino scattering to $\sin ^{2} \theta_{W}$ from other processes.

Neutrino-electron scattering cross sections are sensitive to the existence of additional Z-bosons. It is, however, not possible to define a universal mass-limit valid for all models which predict additional $Z$-bosons, since the coupling and the mass depends on the details of the specific model. A way to gauge limits on additional $Z$-bosons is described in [14]. With the definition, $\Delta g_{A}=g_{A}^{\nu e}-g_{A}$ and $\Delta g_{V}=g_{V}^{\nu e}-g_{V}$, one can write for a coupling $g^{\prime}$ :

$$
m_{Z^{\prime}}^{2}=\left[\frac{g^{\prime}}{g}\right]^{2} m_{Z}^{2} \frac{10}{3} \Delta g_{A} \quad \text { and } \quad m_{Z^{\prime}}^{2}=\left[\frac{g^{\prime}}{g}\right]^{2} m_{Z}^{2} \frac{5}{3} \Delta g_{V}
$$

i.e. the effective coupling constants are changed. 


\section{Experimental methods and results}

As already pointed out, elastic neutrino-electron scattering manifests experimentally as a single forward scattered electron. This signature and the small cross section set the boundary conditions for experimental methods and devices.

Firstly, an intense neutrino beam and a large target mass are required to overcome small event rates. Secondly, good electron identification and reconstruction is essential. Especially an angular resolution in the same order of magnitude as the small scattering angles should be aimed at. Thirdly, the detector should have a large discrimination power against hadron and photon induced backgrounds.

In the following we briefly describe some selected experiments which have contributed in a specific way to our knowledge of neutrino-electron scattering. A more complete description can be found in [15]. Emphasis is put on experimental methods which have evolved with time and increasing number of detected neutrino-electron scattering events.

\section{Muon-neutrino electron scattering}

The study of muon-neutrino electron scattering was only possible when intensive muon-neutrino beams could be provided. At high energy accelerators the source of neutrinos are weak decays of kaons and pions produced by protons in an external target. The mesons are focussed and charge selected by magnetic lenses (horns) [16] before entering a decay region where part of them decay mainly into muons and muon-neutrinos. The surviving hadrons and muons are stopped in massive shieldings. Changing the polarity of the magnetic lenses leads to either neutrino or antineutrino beams. However, muon-neutrino beams are not pure. Due to deficiencies in the meson charge selection and due to $K_{e 3}$ decays contaminations of muon-antineutrinos

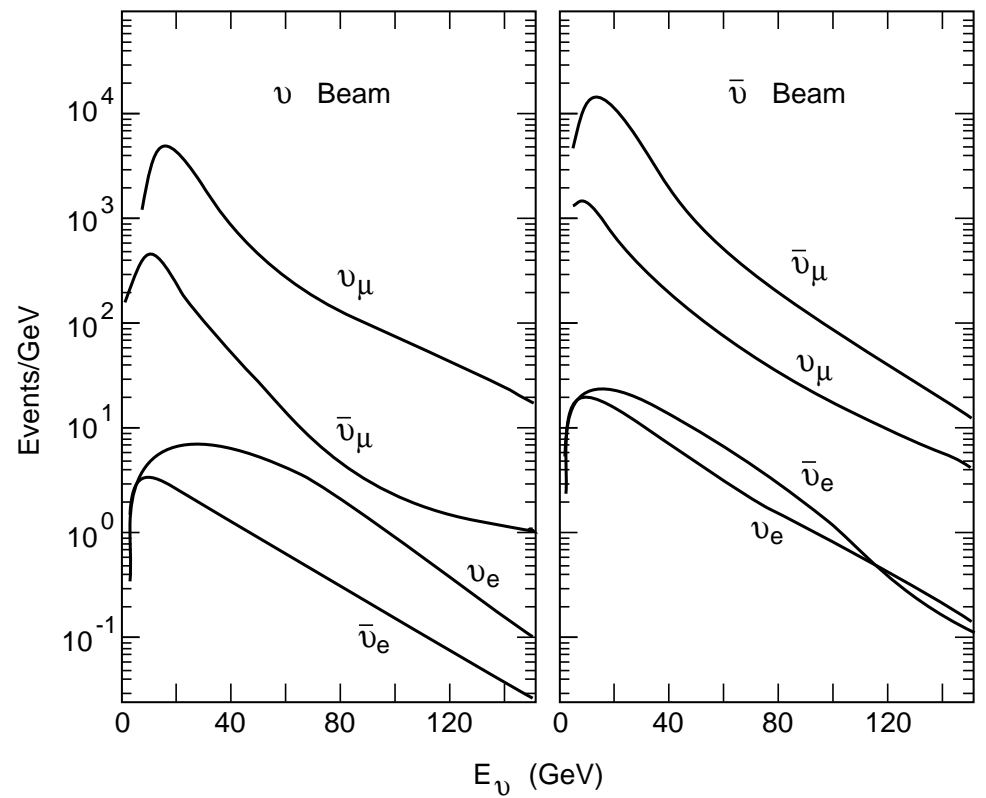

Figure 3: Neutrino energy spectra and beam composition of the CERN $450 \mathrm{GeV} S P S$ wide band neutrino beam. The scales are in arbitrary units. (5-15\%) and electron-(anti)-neutrinos $(\sim 1 \%)$ are present. The CERN SPS wide band neutrino beam (WBB) has a mean energy of $\sim 25 \mathrm{GeV}$ (fig. 3). The neutrino fluxes can be measured by either neutrino induced events for which the cross section is precisely known, or by a measurement of the correlated muon flux in the shielding of the neutrino beam [17].

\section{The Gargamelle experiment}

The first detection of a neutrino-electron-scattering event was achieved in the Gargamelle experiment [18]. This big bubble chamber was exposed to both the CERN PS neutrino beam $\left(\left\langle E_{\nu}\right\rangle \approx 2 \mathrm{GeV}\right)$ and the SPS WBB. The bubble chamber allowed a good particle identification and due to a magnetic field a charge determination. Together with a good angular resolution a powerful background reduction was achieved.

In bubble chambers the main backgrounds are caused by converted $\gamma$-rays from interactions outside the chamber or from bremsstrahlung by muons, quasi-elastic reactions of electron-neutrinos, and neutral pion production. 
In total three (including the historical first NC event) $\bar{\nu}_{\mu} e$ - and ten $\nu_{\mu} e$-events could be detected. This allowed a first determination of the two cross sections, listed in tab. 3. Given the large errors the result was well in agreement with the prediction of the, at that time, young combined electroweak theory.

To increase statistics a new approach was needed. The task was to increase the target mass while keeping the ability to detect and reconstruct electrons.

\section{The CHARM experiment}

In 1977 the CHARM collaboration had assembled a massive $(\sim 100$ tons $)$ counter experiment, initially intended to study semi-leptonic neutrino interactions at the CERN narrow band beam (NBB) [19]. The detector, however, was well suited to study also neutrino-electron scattering. To achieve higher rates it was also exposed to the wide-band neutrino beam (WBB). It consisted of a target-calorimeter followed by a muon-spectrometer. The calorimeter, built from 78 marble plates interspaced with scintillation and proportional counters, combined calorimetry and tracking, and allowed a good discrimination between electromagnetic and hadronic showers. The dominant background to neutrino-electron scattering in this energy domain is coherent and diffractive $\pi^{0}$ production, resulting in an electromagnetic shower hardly distinguishable from a shower induced by an electron. Quasi-elastic scattering of electron-neutrinos on nucleons

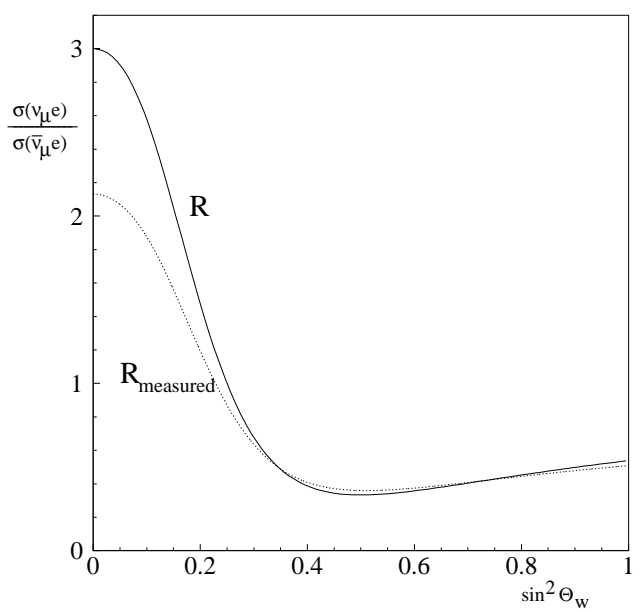

Figure 4: Ratio of cross sections as a function of $\sin ^{2} \theta_{W}$. contribute to a smaller extend to the background. The background is characterized by a much broader distribution in the scattering angle than neutrinoelectron scattering (eqn. 1) which is made use of when subtracting the background statistically.

In two data taking periods $(1979-81,83)$ in total $83 \pm 16 \nu_{\mu} e$ - and $(116 \pm 21) \bar{\nu}_{\mu} e$-events were found [20] leading to measurements of the absolute cross sections for both neutrino and antineutrino electron scattering, (tab. 3). Statistical and systematical errors contribute equally to the total uncertainty. The largest systematic error sources were the neutrino flux normalization and acceptance corrections.

To extract the electroweak mixing angle from their data the CHARM collaboration had invented a new method using the cross section ratio:

$$
R=\frac{\sigma\left(\nu_{\mu} e\right)}{\sigma\left(\bar{\nu}_{\mu} e\right)}=\frac{1+\eta+\eta^{2}}{1-\eta+\eta^{2}} \quad \text { with } \quad \eta=1-4 \sin ^{2} \theta_{W}
$$

which has a large sensitivity to $\sin ^{2} \theta_{W}$ in the region of interest $\left(\Delta \sin ^{2} \theta_{W}=1 / 8 \Delta R\right.$, fig. 4$)$. Sytematic errors cancel largely in this ratio and no absolute knowledge of the neutrino fluxes and selection efficiencies is required. In practice the sensitivity of $R$ to $\sin ^{2} \theta_{W}$ is weakened (fig. 4) due to admixtures of different types of neutrinos in the beam and experiment dependent kinematical cuts. Compared to earlier determinations of $\sin ^{2} \theta_{W}$ from single cross section measurements this technique resulted in a largely reduced error on $\sin ^{2} \theta_{W},($ tab. 3 ).

\section{The experiment E734 at BNL}

The experiment E734 was performed at the Brookhaven National Laboratory (BNL). The neutrino source was the focused wide band neutrino (antineutrino) beam at the AGS (Alternating Gradient Synchroton), with a mean neutrino energy of $1.3 \mathrm{GeV}$. 
The detector design aimed at good particle identification, high angular resolution and a large total mass (170 tons) [21]. The main part of the detector was formed by a target calorimeter followed by a gamma catcher and a muon spectrometer. The calorimeter consisted of 112 planes of liquid scintillator, and two planes of proportional drift tubes. The module thickness of only $0.22 X_{0}$ provided a precise $d E / d x$ measurement and made it possible to separate between electrons and photons and to reduce the $\gamma$-induced background. An excellent angular resolutions for electrons of $\sigma\left(\theta_{\text {proj }}\right)=$ $\left((13 \pm 1) \sqrt{E_{e} / G e V}\right.$ mrad) could be achieved.

Data were taken in three running periods from 1981 to 1986. After background subtraction a total of $N(\nu e)=160 \pm 17_{\text {stat }} \pm 4_{\text {syst }}$ and $N(\bar{\nu} e)=97 \pm 13_{\text {stat }} \pm 5_{\text {syst }}$ were found in the data sample [22]. To obtain results on absolute cross sections and on the cross section ratio these numbers are corrected for wrong-type neutrino contaminations using the current world average for $\sin ^{2} \theta_{W}$.
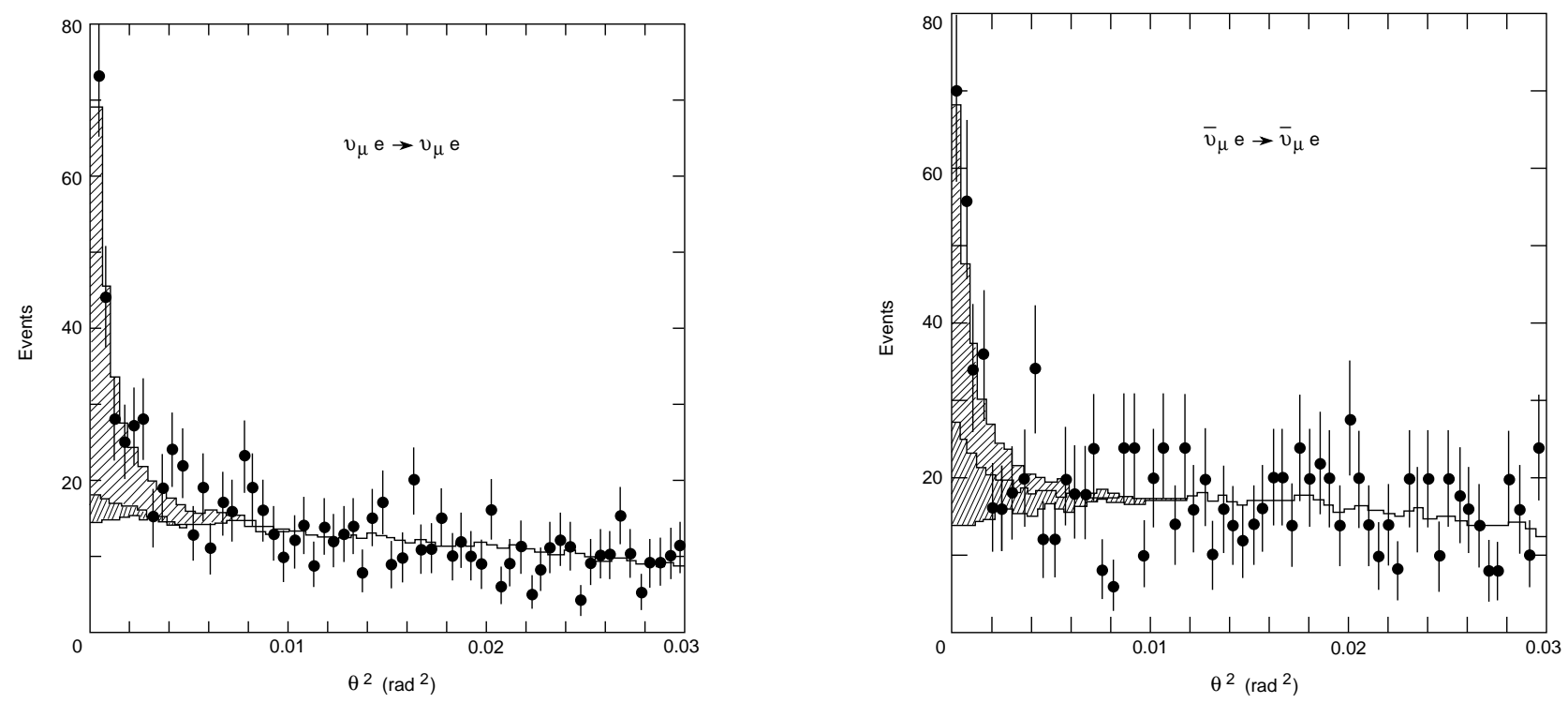

Figure 5: Experimental data and the result of the best fit for the BNL E734 experiment. Data are plotted against $\theta_{e}^{2}$ as full circles and the fit results are displayed as a line. The $y$-independent term is light-shaded (upper part); the $(1-y)^{2}$-term is dark-shaded (lower part).

As the angular resolution of this experiment was excellent a first attempt to explore the information contained in the differential cross sections was made. For that the signal was decomposed into two $y$-dependent components, corresponding to the left-handed and right-handed terms in the expression for the differential cross sections (eqn. 4). The contributions from the wrong helicity components and the electron-neutrinos in the beam were neglected. The data are shown in fig. 5 . Values of the electroweak couplings $g_{V}$ and $g_{A}$ were obtained by fitting the following function to the $\theta^{2}$ distributions of the data:

$$
n_{i}=\frac{G_{F}^{2} m_{e}}{2 \pi} \Phi^{\nu}\left\langle E_{\nu}\right\rangle\left(\left(g_{V} \pm g_{A}\right)^{2} A_{1} f_{i}+\left(g_{V} \mp g_{A}\right)^{2} A_{2} \frac{y_{i}}{3}\right)+N_{b} b_{i}
$$

The index $i$ runs over the $\theta^{2}$ bins. The distributions $f_{i}$ and $y_{i}$ correspond to the $y$-independent and $(1-y)^{2}$-dependent term, respectively, weighted by acceptance functions $A_{k}$, and $N_{b} b_{i}$ is the absolute number of background events.

This method shows a slightly better sensitivity to electroweak parameters and provides results with superior accuracy than those from the absolute cross sections.

\section{The CHARM II experiment}

In 1987 the CHARM II experiment at the CERN-SPS WBB started to take data, with the aim of increasing the number of observed $\nu_{\mu}-e$ events by an order of magnitude compared to previous 
experiments and thus performing a high precision measurement of $\sin ^{2} \theta_{W}$. The original idea again, as in CHARM, was to use the ratio of absolute cross sections for $\nu_{\mu} e$ - and $\bar{\nu}_{\mu} e$-scattering. Later also other methods were developed. In particular the most precise value of $\sin ^{2} \theta_{W}$ was achieved by combining the information of measured distributions of kinematical variables with the pure ratio method to a measurement of the ratio of differential cross sections. The last period of data taking for CHARM II ended in August 1991.

The CHARM-II detector [23] consisted of a fine-grained, massive target calorimeter followed by a muon spectrometer. The target calorimeter was composed of 420 equal units with a total target mass of 600 tons. Each unit consisted of a $5 \mathrm{~cm}$ ( 0.5 radiation length) thick glass plate followed by a plane of streamer tubes, which were read out directly on the wire in a digital mode as well as in an analog mode through pick up strips glued to the back of the tubes. The high granularity of active elements and the low $\mathrm{Z}$ of the target material ensure a good angular resolution and together with the fine sampling a reliable distinction of electromagnetic and hadronic showers. The energy resolution for electrons was found to be $\Delta E / E=0.23 / \sqrt{E / \mathrm{GeV}}+0.05$. The angular resolution was equivalent to $\Delta \theta_{\text {proj }} \approx 17 \mathrm{mrad} / \sqrt{E / \mathrm{GeV}}$ in the energy range of the analysis [24].

The ratio of the neutrino and antineutrino fluxes was obtained by five different methods, four of which were measurements of event-rates of processes with a known cross section ratio for neutrinos and antineutrinos. The ratio of the muon fluxes in the shielding downstream of the decay region yielded a fifth, independent determination. After combining the five consistent results a precision on the flux ratio of $2.2 \%$ was reached. The absolute normalization of the neutrino flux was obtained from inclusive neutrino-nucleon scattering used as a monitor reaction. The total uncertainty on the absolute flux measurement was found to be about $5 \%$.

To explain the idea of the analysis we start with the cross section for $\nu e$-scattering in a general form:

$$
\frac{\mathrm{d} \sigma}{\mathrm{d} y}=\frac{2 G_{F}^{2} m_{e}}{\pi} E_{\nu} \sum_{i=1}^{3} A_{i} g_{i} \quad \text { with } \quad g_{1}=g_{L}^{2}, g_{2}=g_{R}^{2}, g_{3}=\left(g_{L}+1\right)^{2} .
$$

The expressions $A_{i}$ are given in tab. 1 for the four processes involved. The interference of neutral and charged currents in electron-neutrino electron scattering is accounted for in the third term in eqn. 12.

Table 1: Terms of the differential cross section for different processes.

\begin{tabular}{cllll}
\hline \hline Process & $\nu_{\mu} e \rightarrow \nu_{\mu} e$ & $\bar{\nu}_{\mu} e \rightarrow \bar{\nu}_{\mu} e$ & $\nu_{e} e \rightarrow \nu_{e} e$ & $\bar{\nu}_{e} e \rightarrow \bar{\nu}_{e} e$ \\
\hline$A_{1}$ & 1 & $(1-y)^{2}$ & 0 & 0 \\
$A_{2}$ & $(1-y)^{2}$ & 1 & $(1-y)^{2}$ & 1 \\
$A_{3}$ & 0 & 0 & 1 & $(1-y)^{2}$ \\
\hline \hline
\end{tabular}

The measured event rates are given by:

$$
\mathrm{d} n^{\nu e} / \mathrm{d} y=\Phi^{\nu} \sum_{i=1}^{3} f_{i}^{\nu e} g_{i}+\sum_{i=1}^{4} b_{i} f_{i}^{B G}
$$

where the differential distributions $f_{i}^{\nu e}$ contain all information about the target density, energy spectra and wrong component contamination of the beam, the cross section expressions $A_{i}$, and the experimental resolutions and acceptances. All these quantities are either known by calculation or measured. $\Phi^{\nu}$ is a normalization factor related to the neutrino flux. The $f_{i}^{B G}$ stand for the different background distributions that are due to semileptonic processes with dominantly electromagnetic final states. In order of decreasing importance these are: coherent or diffractive $\pi^{0}$-production, quasielastic scattering 
of electron-neutrinos on nuclei and inclusive $\nu N$-scattering. The $b_{i}$ denote the relative abundance of these processes.
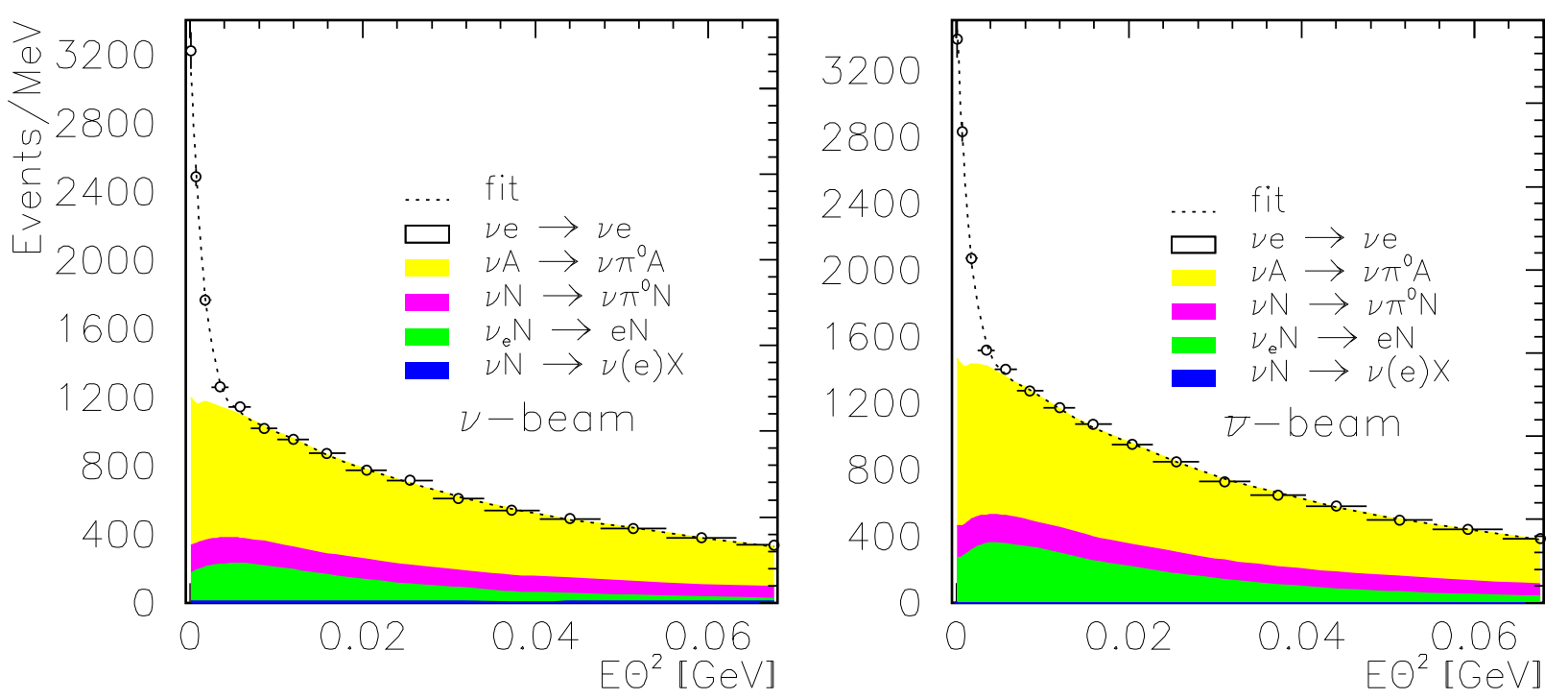

Figure 6: Experimental data and the result of the best fit in the $E_{e} \theta_{e}^{2}$ projection: data are shown as circles and the fit results are displayed as a dashed line.

Electroweak parameters are obtained from a simultaneous fit of modelled differential distributions $f_{i}^{\nu e}$ to the data collected in the $\nu$ - and $\bar{\nu}$ beam. Since the energy of the neutrino is unknown $y$ cannot be measured directly but instead the $f_{i}^{\nu e}$ are double differential distributions in the variables $E_{e} \theta_{e}^{2}$ and $E_{e}$. They discriminate between signal and background in the variable $E_{e} \theta_{e}^{2}$, and determine the background composition because of their different energy $\left(E_{e}\right)$ distributions.

The different analyses described in the following, all based on these differential distributions, mainly differ in the treatment of the factors $\Phi^{\nu}$.

Making use of the absolute flux determination, i.e. fixing $\Phi^{\nu}$ and $\Phi^{\bar{\nu}}$, allows to determine the two coupling constants $g_{V}$ and $g_{A}$ (or $\sin ^{2} \theta_{W}$ and $\rho$ ) simultaneously. The sensitivity of this analysis to $g_{V}$ and $g_{A}$ is illustrated in fig. 7, where the allowed regions in the $g_{V}-g_{A}$ plane are shown, as obtained from the fit.

Due to the electron-neutrino abundance in the

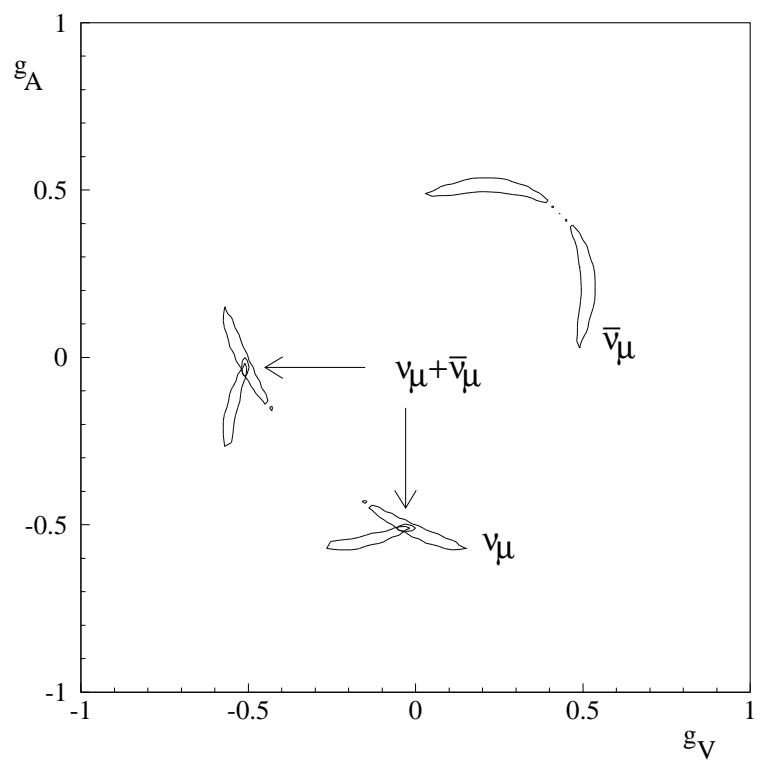

Figure 7: 90\% confidence level contours in the $g_{V}-g_{A}$ plane, as obtained by CHARM II. Only statistical errors are considered. beam the expected fourfold ambiguity is reduced to a twofold one. The CHARM II result, shown in fig. 6 [3], is based on about $2200 \nu$ e-events recorded in each channel. The systematic error is dominated by uncertainties of the background determination, of the neutrino flux measurement and the event selection efficiency.

The most precise determination of $\sin ^{2} \theta_{W}$ results from a similiar fit but uses only the knowledge of the relative neutrino flux. This method is equivalent to the use of the ratio of cross sections, explained above, with the extension that now differential cross sections are used. Also here the systematic error 
is dominated by the background determination. An improved result from the full CHARM II data sample (87-91) is expected to be published soon.

Table 2: Summary of results of the CHARM II experiment.

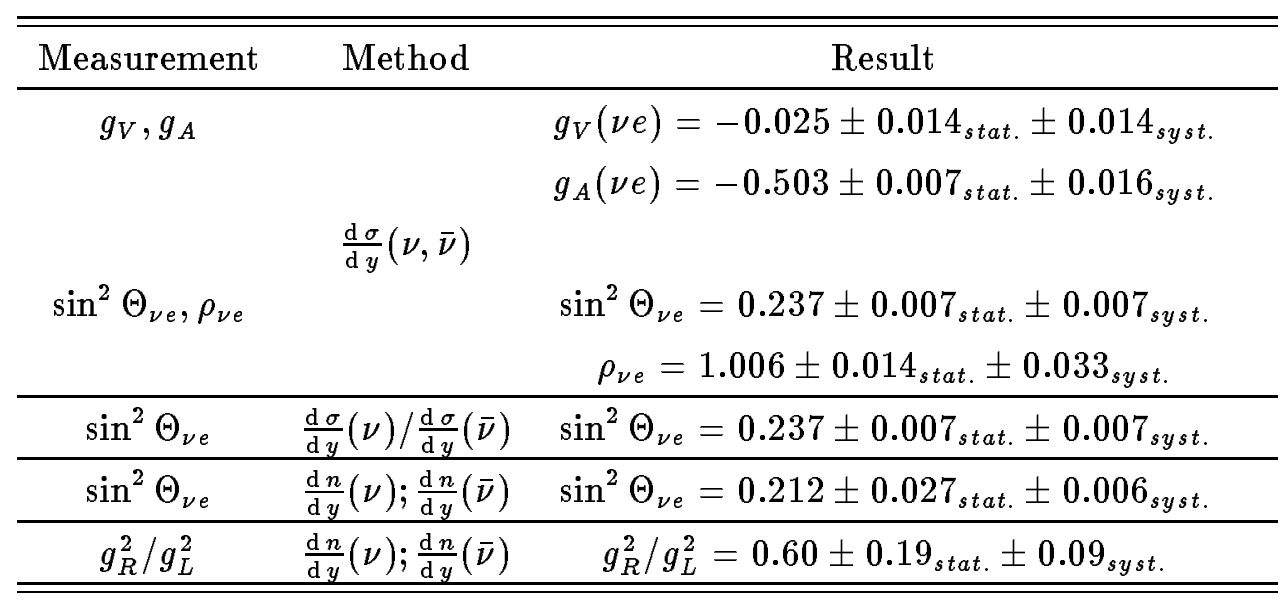

The large statistics accumulated made it possible for the first time to use the information of the shapes of kinematical distributions [25] only. $\sin ^{2} \theta_{W}$ is obtained by another fit in the variables $\left(E_{e} \theta_{e}^{2}, E_{e}\right)$, leaving both parameters $\Phi^{\nu, \bar{\nu}}$ free, hence assuming no knowledge on the neutrino flux.
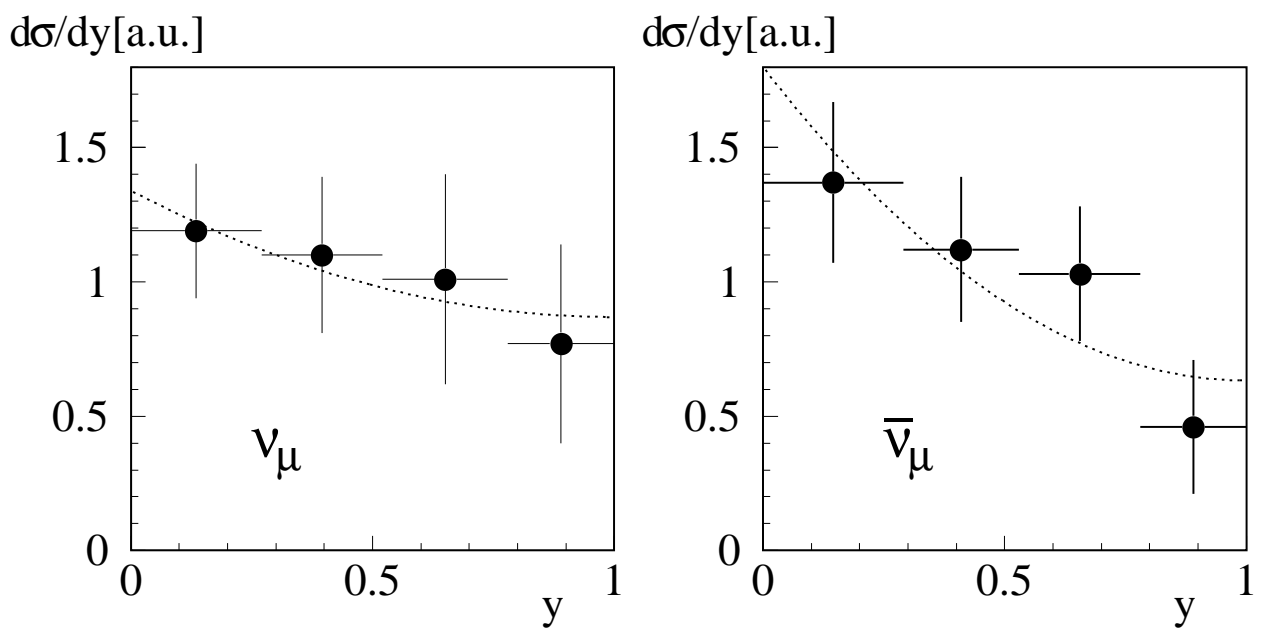

Figure 8: Differential cross sections for $\nu_{\mu}$ e-scattering (left), and $\bar{\nu}_{\mu}$ e-scattering (right) in arbitrary units. Only statistical errors are shown. The dashed line corresponds to the prediction of the Standard Model for a value of the electroweak mixing angle of $\sin ^{2} \theta_{W}=0.212$.

An independent analysis, which is again using only shapes of kinematical distributions is the regularized unfolding [26] of the $y$-distributions for $\nu_{\mu} e$ and $\bar{\nu}_{\mu} e$-scattering from the measured distributions of the variables $\left(E_{e} \theta_{e}^{2}, E_{e}\right)$. The unfolded cross sections are shown in fig. 8. A fit of the Standard Model prediction for the differential cross sections determines the ratio of coupling constants $\left(g_{R}^{2} / g_{L}^{2}\right)$ (tab. 2) [25]. The main contribution to the systematic error is the uncertainty on the absolute energy scale. The result confirms the existence of a coupling of right-handed electrons to the $Z^{0}$ by three standard deviations and by this non-maximal parity violation in neutral current interactions. 


\section{Electron-neutrino electron scattering}

Compared to the muon-neutrino sector the number of experimental results in electron-neutrino electron scattering is still rather poor. This has two reasons, firstly, pure electron-neutrino beams are more difficult to produce than muon-neutrino beams, and secondly $\nu_{e^{-}}$and $\bar{\nu}_{e}$-beams are much lower in energy, so that large event rates are difficult to achieve.

Electron-antineutrino electron scattering was first observed in the Savannah River Reactor experiment [27]. The fission reactor produced a high flux of $\bar{\nu}_{e}$. The data were analysed in two energy regions and gave results consistent with the Standard Model (tab. 3).

\section{The experiment E225 at LAMPF}

A dedicated experiment to search for $\nu_{e} e$-scattering was performed at the Los Alamos Meson Physics Facility (LAMPF) [28]. It aimed at a measurement of the $\mathrm{NC}-\mathrm{CC}$ interference term in $\nu_{e} e$-scattering.

An elegant way of producing a $\nu_{e}$-enriched neutrino beam was performed at LAMPF. Starting with an $800 \mathrm{MeV}$ proton beam, pions are produced, stopped, and decay at rest producing an isotropic flux of monochromatic muon-neutrinos $(30 \mathrm{MeV})$. In the subsequent muon decay a continious spectrum of $\nu_{e}$ and $\bar{\nu}_{\mu}$ (fig. 10) is produced. The spectra can easily be calculated, however, the determination of the absolute neutrino flux requires some dedicated calibration experiments.

The detector consisted of a 15 ton central active target, segmented into 40 scintillator planes interspaced by multi-plane flash chamber modules. The scintillators were used to measure the energy loss, while the flash chambers determined position and direction of the particles. The main background sources are cosmic rays, neutron capture, and $\nu_{e} C$ reactions.

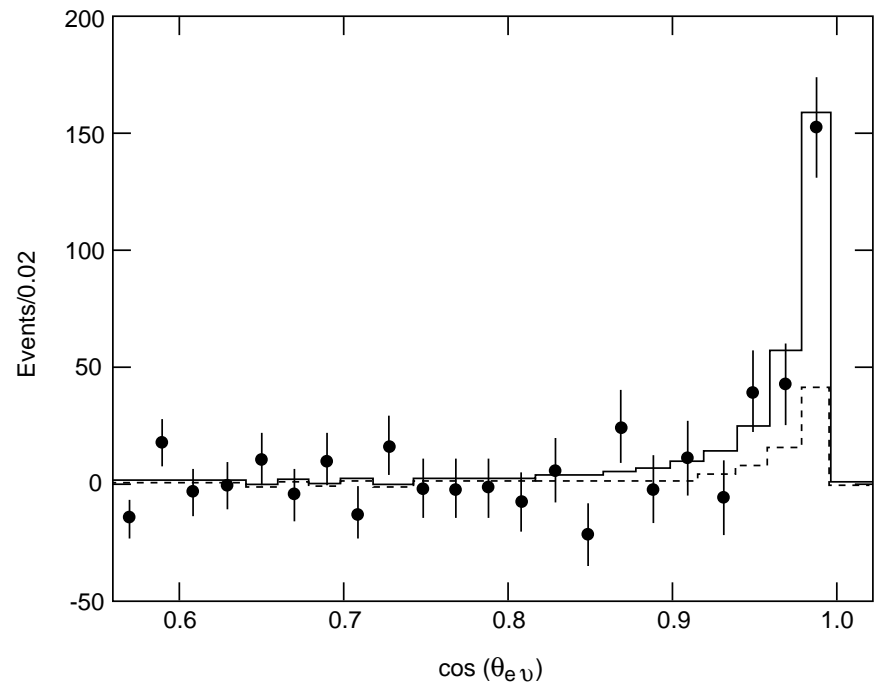

Figure 9: Angular distribution of the measured vesignal in E225. The solid line is the result of the best fit, $N(\nu e)=295 \pm 35$ events. The dashed line is the background contribution due to $\nu_{\mu}$ e and $\bar{\nu}_{\mu} e$.

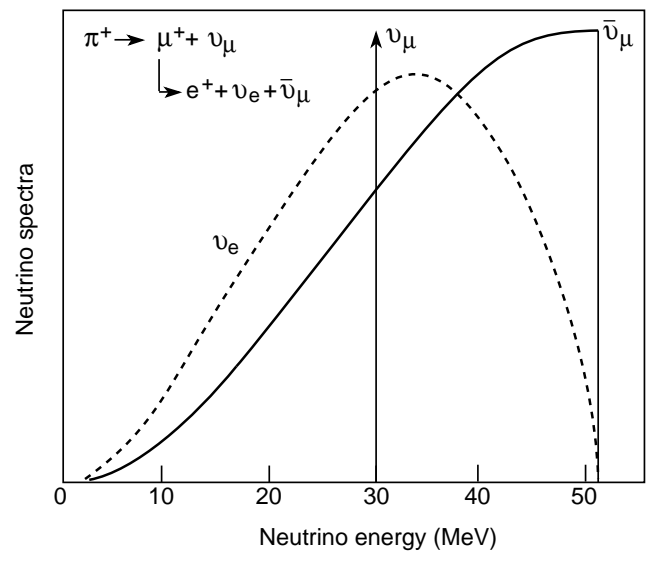

Figure 10: Energy spectrum of the $L A M P F$ neutrino beam produced by pion and muon decay at rest.

The $\nu e$-signal was extracted from the data by a fit to the distributions of the recoil energy $E_{e}$ and angle $\cos \theta_{e}$ (fig. 9). After subtracting the contributions from $\nu_{\mu} e$ and $\bar{\nu}_{\mu} e$-scattering, using measured cross sections, $236 \pm 35 \nu_{e} e$-events were found from which a total cross section measurement was derived [29].

The result was interpreted in terms of the $\mathrm{NC}-\mathrm{CC}$ interference. The cross section for electron- 
neutrino electron scattering can be written as:

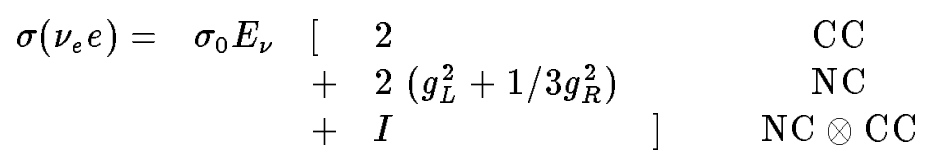

where $I$ denotes the interference term. After subtraction of the pure $\mathrm{NC}$ and $\mathrm{CC}$ contributions destructive interference was found:

$$
I=-1.07 \pm 0.17_{\text {stat }} \pm 0.11_{\text {syst }}
$$

in very good agreement with the Standard Model prediction of:

$$
I=4 g_{L}=-2+4 \sin ^{2} \theta_{W}=-1.07 \quad \text { with } \quad \sin ^{2} \theta_{W}=0.233 .
$$

Profitting from the low neutrino energy a limit on a magnetic moment of the neutrino derived from neutrino electron scattering could be presented [30]:

$$
\mu_{\nu}<0.61 \times 10^{-9} \mu_{\text {Bohr }}(90 \% \text { C.L. })
$$

This is, at present, the best limit from either reactor or accelerator experiments. It has to compete with limits derived from astrophysical arguments. Those are more stringent by about three orders of magnitudes, but on the other hand relying on many more theoretical assumptions.

\section{Discussion}

In tab. 3 results from all neutrino-electron scattering experiments are summarized. A few comments are necessary. All quoted cross section results are model-dependent. This has the following reasons. The raw event rates have to be corrected for the limited kinematical acceptance of the experiment and for the admixture of events originating from other neutrino types. For these corrections a model has to be assumed. This is normally the Standard Model, however with different values for $\sin ^{2} \theta_{W}$. This creates no problem as long as the corrections, which can be as large as 10-20\%, are much smaller than the experimental error. However, for precision measurements it is preferable to measure directly $g_{V}$ and $g_{A}$ rather than the cross sections. This is almost model-independent since any admixture of $V$ and $A$ terms is allowed, and, even more important, all neutrino species can be treated in the same way, hence no correction has to be applied. This procedure was applied by the CHARM II experiment, but the fact that it is not used by all experiments makes a combination off results difficult.

Nevertheless an attempt was made to combine all results listed in tab. 3, taking into account possible correlations between the single results. A $\chi^{2}$-fit was performed with the neutral current coupling constants $g_{V}$ and $g_{A}$ as free parameter. The result is:

$$
g_{V}(\nu e)=-0.034 \pm 0.016_{\text {total }} \quad \text { and } \quad g_{A}(\nu e)=-0.504 \pm 0.014_{\text {total }}
$$

with a correlation coefficient of 0.05 . The quality of the fit is good $\left(\chi^{2}=7.7 / 14\right)$.

The result is shown in fig. 11 together with results of individual experiments seperately for $\stackrel{(-)}{\nu}_{e^{-}}$ and $\stackrel{(-)}{\nu}_{\mu}$-experiments. Obviously the precision of the combined results is dominated by the CHARM II result.

Using relation (3) and (5) the result can be expressed in terms of the electroweak mixing angle and the relative coupling strength of the neutral and charged current:

$$
\sin ^{2} \theta_{\nu e}=0.233 \pm 0.008_{\text {total }} \text { and } \rho_{\nu e}=1.007 \pm 0.028_{\text {total }} .
$$

The correlation factor is 0.09 . 
The high precision on electroweak parameters achieved now in neutrino-electron scattering experiments makes it interesting to compare these results to high precision results obtained in experiments studying different processes.

At LEP, for instance, the annihilation process $e^{+} e^{-} \rightarrow l \bar{l}$ via $Z^{0}$ exchange is studied, whose diagram is related by crossing symmetry to the one of $\nu e$-scattering. However, the two measurements refer to different $Q^{2}$ scales. Differences of the two couplings at the two scales $\left(Q^{2}=0.01 \mathrm{GeV}^{2}\right.$ and $Q^{2}=m_{Z}^{2}$ ) are expected to arise from the running of the fine structure constant $\alpha$ and the effect of the neutrino charge radius. By chance these different contributions numerically cancel almost completely, resulting in a difference of $g_{V}(\nu e)-g_{V}(L E P)=-0.002$, while the individual contributions to radiative corrections are larger by an order of magnitude [9]. Both contributions depend on the masses of the top quark and of the Higgs boson. For the calculation the masses had been fixed to $m_{\text {top }}=150 \mathrm{GeV}$ and $m_{H}=100 \mathrm{GeV}$. Thus it is possible to compare $\nu e$-results directly to those obtained from a measurement of the partial width $\Gamma_{l l}$ (assuming lepton universality) at the $Z^{0}$ resonance and the forward-backward asymmetry $A^{F B}$ at LEP.

Fig. 12 shows this comparison. Remarkable is that results from neutrino-electron scattering have reached comparable precision in $g_{V}^{e}$ and the excellent agreement of measurements over a $Q^{2}$-range of

Table 3: Compilation of total cross section and $\sin ^{2} \theta_{\nu e}$ measurements for all neutrino-electron scattering experiments. Limits are given at the 90\% C.L.

\begin{tabular}{|c|c|c|c|c|}
\hline \multicolumn{2}{|l|}{ Experiment } & \multirow{2}{*}{\multicolumn{2}{|c|}{$\begin{array}{c}\sigma\left(\nu_{\mu} e\right) / E_{\nu} \\
\left(\times 10^{-45} \mathrm{~cm}^{2} \mathrm{MeV}^{-1}\right)\end{array}$}} & \multirow{2}{*}{$\sin ^{2} \theta_{\nu e}$} \\
\hline & & & & \\
\hline Gargamelle (PS) & {$[18]$} & $<1.4$ & $1.0_{-0.9}^{+2.1}$ & $0.1<x<0.4$ \\
\hline Aachen-Padova (PS) & {$[31]$} & $1.1 \pm 0.6$ & $2.2 \pm 1.0$ & $0.35 \pm 0.08$ \\
\hline Gargamelle (SPS) & {$[18]$} & $2.4_{-0.9}^{+1.2}$ & $<2.7$ & $\mathbf{0 . 1 2} 2_{-0.07}^{+0.11}$ \\
\hline VMWOF (FNAL) & {$[32]$} & $1.4 \pm 0.3 \pm 0.4$ & & $0.25_{-0.05}^{+0.07} \pm 0.8$ \\
\hline BNL-COL (AGS) & {$[33]$} & $1.67 \pm 0.44$ & & $0.20_{-0.05}^{+0.06}$ \\
\hline 15-feet BC (FNAL) & {$[34]$} & & $<2.1$ & $<0.37$ \\
\hline BEBC-TST (SPS) & {$[35]$} & & $<3.4$ & $<0.45$ \\
\hline CHARM (SPS) & {$[20]$} & $2.2 \pm 0.4 \pm 0.4$ & $1.6 \pm 0.3 \pm 0.3$ & $0.211 \pm 0.035 \pm 0.011$ \\
\hline BNL E734 (AGS) & {$[22]$} & $1.8 \pm 0.2 \pm 0.25$ & $1.17 \pm 0.16 \pm 0.13$ & $0.195 \pm 0.018 \pm 0.013$ \\
\hline \multirow[t]{3}{*}{ CHARM-II (SPS) ${ }^{\dagger}$} & {$[3]$} & $1.53 \pm 0.04 \pm 0.12$ & $1.39 \pm 0.04 \pm 0.10$ & $0.237 \pm 0.007 \pm 0.007$ \\
\hline & & $\sigma\left(\nu_{e} e\right) / E_{\nu}$ & $\sigma\left(\bar{\nu}_{e} e\right)$ & \\
\hline & & $\left(\times 10^{-42} \mathrm{~cm}^{2} \mathrm{GeV}^{-1}\right)$ & $\left(\times 10^{-46} \mathrm{~cm}^{2}\right)$ & \\
\hline Savannah River & {$[27,36]$} & & $7.6 \pm 2.2^{a}$ & $0.25 \pm 0.05$ \\
\hline (Reactor) & & & $1.86 \pm 0.48^{b}$ & \\
\hline Kurchatov (Reactor) $)^{\frac{\dagger}{t}}$ & {$[37]$} & & $6.8 \pm 4.5$ & $0.29 \pm 0.10$ \\
\hline LAMPF E225 (LAMPF) & {$[29]$} & $10.0 \pm 1.5 \pm 0.9$ & & $0.249 \pm 0.063$ \\
\hline
\end{tabular}

$\dagger$ The result on the cross section was derived from the published result on the coupling constants.

$\stackrel{\dagger}{\dagger}$ Preliminary result.

${ }^{a}$ Region in visible energy: $[1.5 . .3 .0] \mathrm{MeV}$

${ }^{b}$ Region in visible energy: [3.0..4.5] $\mathrm{MeV}$ 

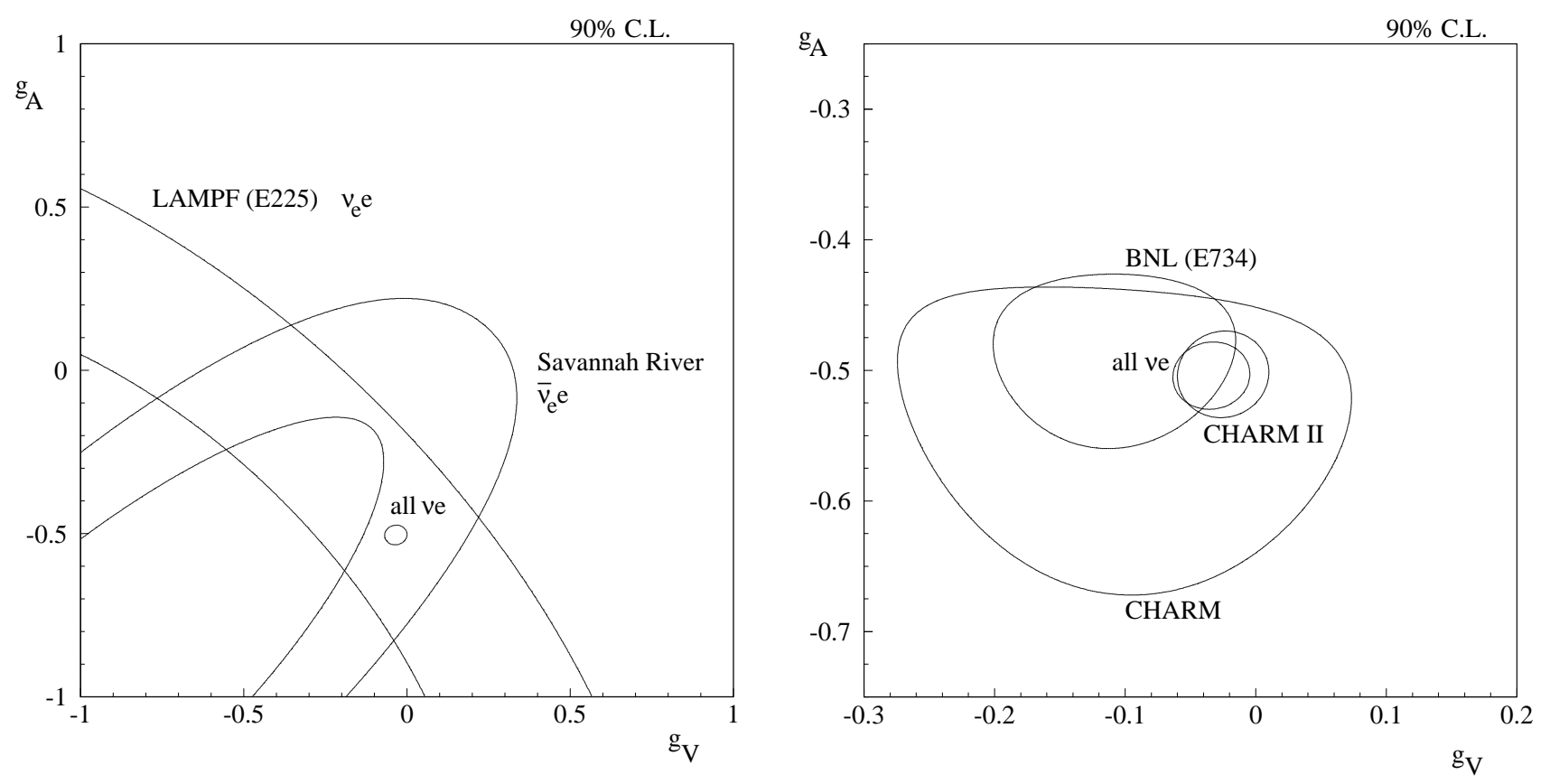

Figure 11: Left: Comparison of results from $\nu_{e} e$ and $\bar{\nu}_{e}$ escattering and the result of the fit to all $\nu_{\mu(e)} e^{-}$ data in the $g_{V}-g_{A}$ plane. Right: Comparison of the most recent $\nu_{\mu}$ e-scattering experiments and the fit to all data. From the solutions of the $\nu_{\mu} e$ and $\bar{\nu}_{\mu} e$ data the ones with $g_{V} \approx-0.5$ or $g_{A} \approx-0.5$ are selected by $\nu_{e} e$ and $\bar{\nu}_{e} e$ experiments. The solution $g_{V} \approx-0.5$ is excluded by $e^{+} e^{-}$data.

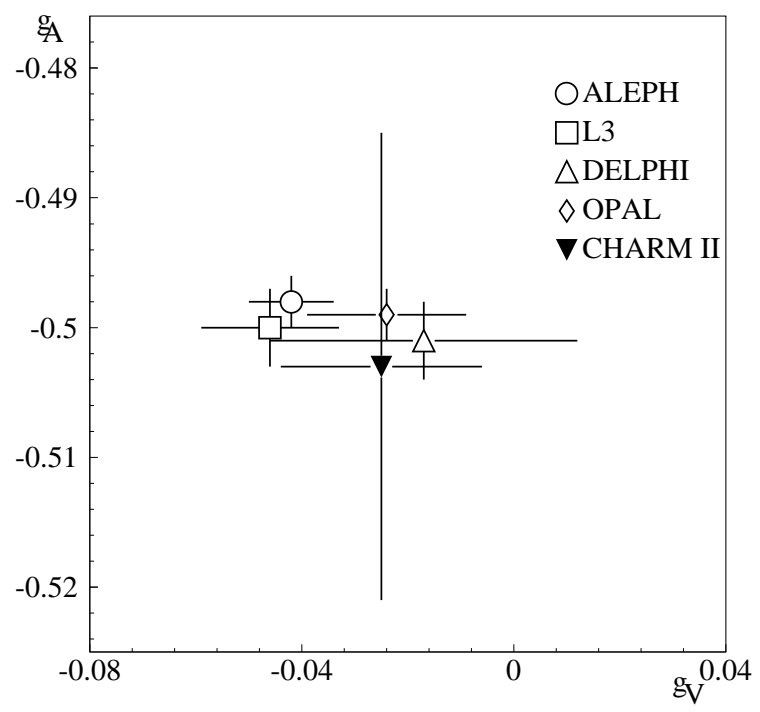

Figure 12: Comparison of results from neutrino-electron scattering and $e^{+} e^{-} \rightarrow l^{+} l^{-}$annihilation at the $Z^{0}$ pole in the $g_{V}-g_{A}$ plane. The crosses show different experimental data points [6, 3].

six orders of magnitude.

As was demonstrated first by the CHARM experiment [20] the combination of $e^{+} e^{-}$and $\nu e$-results selects $g_{A}=-1 / 2$, confirming the prediction from the doublet structure of fermions in the Standard Model.

The coupling constants $g_{V}$ and $g_{A}$, measured in $\nu e$-scattering are in fact a product of the neutrino coupling to the $Z$ and the electron coupling to the $Z$ [38]. The same parameters measured in the process $e^{+} e^{-} \rightarrow e^{+} e^{-}$are sensitive to the $e-Z$ coupling, $g_{V}^{e}$, only. The measurement of the invisible width of the $Z$ at LEP determines the coupling to a mixture of all three neutrino species. When we 
use the notation $g_{V}^{\nu e}$ and $g_{A}^{\nu e}$ for the coupling measured in $\nu e$-scattering, one finds

$$
g_{V, A}^{\nu e}=2 g^{\nu} g_{V, A}^{e}
$$

where $g^{\nu}$ is the $\nu-Z$ coupling, predicted by the weak isospin structure of the Standard Model to be

$$
g^{\nu}=g_{V}^{\nu}=g_{A}^{\nu}=1 / 2
$$

If no assumption of lepton universality is made, the combination of LEP-data and the CHARM-II data gives:

$$
\left|2 g^{\nu_{\mu}}\right|=1.006 \pm 0.036 .
$$

Hence $\nu_{\mu} e$-data provide a unique measurement of the muon-neutrino coupling, while the LEP-result from the invisible width yields $[6,38]$ :

$$
\left|2 g^{\nu_{1}}\right|=1.006 \pm 0.006
$$

for the mixture of the three contributing neutrinos. The fact that both numbers agree among each other confirms lepton universality in the neutrino sector for neutral-current interactions. The values also confirm the prediction based on the weak isospin structure of the Standard Model.

Since the mass of the $Z^{0}$ is measured precisely at LEP [6] $\sin ^{2} \theta_{W}$ is a derived quantity in the Standard Model. To compare the now rather precise results of neutrino-electron scattering with the predictions of the Standard Model one has to apply higher order corrections (section 1). Using the $\overline{M S}$ renormalization scheme [8] one can correct the combined result (18) of $\sin ^{2} \theta_{W}$ and $\rho$. The net correction for $\sin ^{2} \theta_{W}$ is negligible and for $\rho$ it is -0.005 , with a theoretical uncertainty of \pm 0.002 and \pm 0.004 , respectively, induced by assuming a reasonable range of masses $\left(m_{\text {top }}=[80 . .180] \mathrm{GeV}\right.$ and $\left.m_{H}=[50 . .1000] \mathrm{GeV}\right)$. Applying these corrections to the combined result (18) one obtains:

$$
\begin{aligned}
\sin ^{2} \bar{\theta}(\nu e) & =0.233 \pm 0.008_{\text {exp. }} \pm 0.002_{\text {theor. }} \\
\bar{\rho}(\nu e) & =1.002 \pm 0.029_{\text {exp. }} \pm 0.004_{\text {theor. }}
\end{aligned}
$$

This result can be compared to the expectation from the Standard Model using as input parameters $\alpha, G_{F}$, and the mass of the $Z^{0}$ as measured at LEP [6]:

$$
\begin{aligned}
& \sin ^{2} \bar{\theta}\left(\alpha, G_{F}, m_{Z}\right)=0.233 \pm \mathbf{0 . 0 0 2} 2_{\text {theor. }} \\
& \bar{\rho}\left(\alpha, G_{F}, m_{Z}\right)=1.001 \pm 0.004_{\text {theor. }} .
\end{aligned}
$$

The agreement is excellent. Due to the relatively large error on the experimental results it is not possible to derive competetive limits on the top quark mass from this comparison alone. In a global fit to all neutral current data, however, results from neutrino-electron scattering contribute significantly [39].

Also limits on contributions from terms beyond the Standard Model description can be be derived. From a comparison of results on the neutral current coupling constants from neutrino-electron scattering (17) and LEP we obtain a limit for the mass of an additional $Z^{\prime}$-boson with the assumption of equal coupling compared to the standard $Z$ :

$$
m_{Z^{\prime}}>462 \mathrm{GeV} \quad(90 \% \text { C.L. })
$$

This limit is competitive to the best published value, obtained in $p \bar{p}$ collisions by the CDF collaboration [40]: $m_{Z^{\prime}}>412 \mathrm{GeV}$ (95\% C.L.).

A limit on the anomalous charge radius of the neutrino is obtained comparing results for the electroweak mixing angle from neutrino-electron scattering given in (18) with LEP measurements of processes $Z \rightarrow l \bar{l}$, where $l, \bar{l}$ are charged leptons $[6]$ :

$$
\left|\left\langle r^{2}\right\rangle_{\text {anom }}\right|<0.40 \times 10^{-32} \mathrm{~cm}^{2} \quad(90 \% \text { C.L. })
$$


The future of dedicated neutrino-electron scattering experiments probing the Standard Model is uncertain. A promising proposal has been presented at LAMPF, aiming for a $1 \%$ measurement of $\sin ^{2} \theta_{W}[42]$. The approval is still pending.

As the process seems to be understood very well neutrino-electron scattering may be used to explore fields where more understanding is needed, e.g. the solar neutrino problem, or the intrinsic properties of neutrinos.

\section{Conclusion}

During twenty years since the discovery of neutral current interactions, neutrino-electron scattering has contributed in a major way to our understanding of the Standard Model. All possible ve-reactions have been observed and studied and recent experiments have collected many thousands of $\nu e$ events.

Experimental results obtained from these experiments are in very good agreement with predictions of the Standard Model and the very precise results from LEP experiments. The weak-isospin structure of the Standard Model has been verified in the neutral-current sector, lepton-universality for neutrinos has been demonstrated in the neutral-current sector, the ability of the Standard Model to describe precision results using higher order corrections has been shown, and once again no deviation from the Standard Model has been found.

\section{Acknowledgements}

Many fruitful discussions with our colleagues in the CHARM II collaboration are kindly acknowledged.

\section{References}

[1] Gargamelle collab., F.J. Hasert et al., Phys. Lett. B 46 (1973) 121, Phys. Lett. B 46 (1973) 138.

[2] S. Weinberg, Phys. Rev. Lett. 19 (1967) 1264;

A. Salam in Elementary Particle Theory, ed. N. Svartholm (Almquist and Wiksells, Stockholm, 1969) 367;

S.L. Glashow, J. Iliopoulos and L. Maiani, Phys. Rev. D 2 (1970) 1285.

[3] CHARM II collab., P. Vilain et al., Phys. Lett. B 281 (1992) 159.

[4] KAMIOKANDE-collab., K.S. Hirata et al., Phys. Rev. D59 (1987) 2604;

T. Kajita et al., ICRR-283-92-21, 1992, presented at $26^{\text {th }}$ Int. Conf. on High Energy Physics, Dallas, TX.

[5] G. t'Hooft, Phys. Lett. B 37 (1971) 195. Phys. Lett. B 276 (1992) 247.

[6] The LEP collaborations: ALEPH, DELPHI, L3 and OPAL, Phys. Lett. B 276 (1992) 247.

[7] S. Sarantakos, A Sirlin and W.J. Marciano, Nucl. Phys. B 217 (1983) 84.

[8] M. Consoli and W. Hollik, in: G. Altarelli et al. (edts), Report CERN 89-08(1989) 7.

[9] D.Yu. Bardin and V.A. Dokuchaeva, Nucl. Phys. B 246 (1984) 221; Preprint JINR E2-86-260 (1986), D.Yu. Bardin, NUFITTER, a program to calculate electroweak radiative corrections for neutrino-electron scattering.

[10] G. Domogatskii and D. Nadezhin, Sov. J. Nucl. Phys. 12 (1971) 678;

A.V. Kyuldjiev, Nucl. Phys. B 243 (1984) 387.

[11] P. Vogel and J. Engel, Phys. Rev. D 39 (1989) 3378;

M.B. Voloshin, M.I. Vysotskii, and L.B. Okun, Zh. Eksp. Teor. Fiz. 91 (1986) 754.

[12] G. Degrassi, A. Sirlin and W.J. Marciano, Phys. Rev. D 39 (1989) 287;

J.L. Rosner, Phys. Rev. D42, 3107 (1990).

[13] J.E. Kim, V.S. Mathur and S. Okubo, Phys. Rev. D 9 (1974) 3050.

[14] J.A. Grifols and S. Peris, Phys. Lett. B 168 (1986) 264;

J.L. Rosner, Comm. Nucl. Part. Phys. 14 (1985) 229. 
[15] J. Panman, Neutrino-Electron Scattering, in: P. Langacker (ed.), Precision Tests of the Standard Electroweak Model, World Scientific, 1993,

K. Winter, Neutrino reactions and the structure of the neutral weak current, in: K. Winter (ed.), Neutrino Physics, Cambridge University Press, 1991, and references therein.

[16] S. van der Meer, CERN 62-16 (1962).

[17] E.H.M Heijne, CERN 83-06 (1986).

[18] Gargamelle collab., J. Blietschau et al., Nucl. Phys. B 114 (1976) 189;

P. Alibran et al., Phys. Lett. B 74 (1978) 422;

N. Armenise et al., Phys. Lett. B 86 (1979) 225;

D. Bertrand et al., Phys. Lett. B 84 (1979) 354.

[19] CHARM collab., A.N. Diddens et al., Nucl. Inst. \& Meth. 176 (1980) 189.

[20] CHARM collab., J. Dorenbosch et al., Z. Phys. C 41 (1989) 567.

[21] L.A. Ahrens et al., Nucl. Inst. EG Meth. A 254 (1987) 515.

[22] L.A. Ahrens et al., Phys. Rev. D 41 (1990) 3297.

[23] CHARM II collab., K. de Winter et al., Nucl. Inst. E Meth. A 278 (1989) 670.

[24] CHARM II collab., D. Geiregat et al., Nucl. Inst. ES Meth.A 325 (1993) 92.

[25] CHARM II collab., P. Vilain et al., Phys. Lett. B 302 (1993) 351.

[26] V. Blobel, DESY 84/118, Hamburg 1984.

[27] F. Reines, H.S. Gurr and H.W. Sobel, Phys. Rev. Lett. 37 (1976) 315.

[28] R.C. Allen et al., Nucl. Inst. \& Meth. A 269(1988) 177.

[29] R.C. Allen et al., Phys. Rev. D 47 (1992) 11.

[30] D.A. Krakauer et al., Phys. Lett. B 252 (1990) 177.

[31] H. Faissner et al., Phys. Rev. Lett. D 41 (1978) 213.

[32] R.H. Heisterberg et al., Phys. Rev. Lett. 44 (1980) 635.

[33] A.M. Cnops et al., Phys. Rev. Lett. 41 (1978) 367;

N.J. Baker et al., Phys. Rev. D 40 (1989) 2753.

[34] J.P. Berge et al., Phys. Lett. B 84 (1979) 357.

[35] BEBC-TST collab., N. Armenise et al., Phys. Lett. B 81 (1979) 385.

[36] F.T. Avignone, III, Phys. Rev. D 2 (1970) 2609.

[37] G.S. Vidyakin et al., JETP Lett. 49 (1989) 740.

[38] V.A. Novikov, L.B. Okun and M.I. Vysotsky, Phys. Lett. B $298(1993) 453$.

[39] J. Ellis, G.L. Fogli and E. Lisi, CERN-TH-6643-92 (1992).

[40] CDF collab., F. Abe et. al., Phys. Rev. Lett. 68 (1992) 1463.

[41] Particle Data Group, Phys. Rev. D45 (1992) 1.

[42] R.C. Allen et al., LANL Proposal, LA-11 300-P, 1988. 